\title{
Polymorphisms of VEGF and VEGF receptors are associated with the occurrence of ovarian hyperstimulation syndrome (OHSS) - a retrospective case-control study
}

Kazem Nouri ${ }^{1}$, Peter Haslinger ${ }^{1}$, Ladislaus Szabo ${ }^{1}$, Michael Sator ${ }^{1,2}$, Martin Schreiber ${ }^{1}$, Christian Schneeberger ${ }^{1}$ and Detlef Pietrowski ${ }^{*}$

\begin{abstract}
Background: Ovarian hyperstimulation syndrome (OHSS) is the most serious complication of IVF/ICSI therapy. The pathophysiology and etiology of the disease is still not fully clarified.

Methods: To assess whether polymorphisms of the VEGF/VEGF-receptor system contribute to the occurrence of ovarian hyperstimulation syndrome (OHSS), we performed a retrospective analysis of 116 OHSS patients, and 124 female controls. The following SNPs were genotyped: Rs2071559 (VEGFR2-604); rs2305948 (VEGFR2-1192); rs 1870377 (VEGFR2-1719); rs2010963 (VEGF-405); and rs111458691 (VEGFR1-519). Odds ratios (ORs) were estimated with a 95\% confidence interval (CI). Linkage disequilibrium (LD) analysis was performed in the three loci of the VEGFR2 gene.

Result: We found an overrepresentation of the T allele of the VEGFR1-519 polymorphism in OHSS patients ( $P=0.02$, OR: 3.62, Cl: 1.16 - 11.27). By genotype modeling, we found that polymorphism of VEGFR1-519 and VEGF-405 showed significant differences in patients and controls $(p=0.02$, OR: $3.79 \mathrm{Cl}: 1.98-11.97$ and $p=0.000005$, OR: 0.29, Cl: 0.17 - 0.50). LD analysis revealed significant linkage disequilibrium in VEGFR2.
\end{abstract}

Conclusion: Polymorphisms in the VEGFR2 gene and in the VEGF gene are associated with the occurrence of OHSS. This strengthens the evidence for an important role of the VEGFNEGF- receptor system in the occurrence of OHSS.

Keywords: ART, IVF-therapy, Vascular permeability, VEGF, OHSS

\section{Introduction}

Iatrogenic ovarian hyperstimulation syndrome (OHSS) is the most serious complication of IVF/ICSI therapy. OHSS is characterized by ovarian enlargement and fluid shift from the intravascular to the extravascular compartment [1]. Although the etiology of OHSS is far from being fully understood, there are two frequently observed pathologies: (i) an increase in vascular permeability; and (ii) HCG is the triggering factor for this condition $[2,3]$.

The mechanism by which HCG triggers vascular permeability in OHSS patients is still under investigation, but the vascular endothelial growth factor (VEGF) and its

\footnotetext{
*Correspondence: detlef.pietrowski@meduniwien.ac.at

'Department of Endocrinology and Reproductive Medicine, Medical

University Vienna, Währinger Gürtel 18-20, 1090 Vienna, Austria

Full list of author information is available at the end of the article
}

receptors seem to play a key role in this physiological process [4-7]. These observations are strengthened by the findings that the probability of developing OHSS correlates with the concentration of VEGF in serum [8-11] and that, in OHSS patients, the amount of VEGF in the follicular fluid is frequently higher than in individuals not affected by this complication [12]. Interestingly, both the clinical pregnancy rate and abortion rate in OHSS patients were significantly higher than that in patients without the syndrome $[13,14]$. These observations suggest that early pregnancy processes, i.e., implantation, trophoblast invasion, or placentation, are also affected in OHSS patients [15].

VEGF triggers its signalling via a set of three main receptors named VEGF receptor 1 to 3 (VEGFR1, VEGFR2, 
VEGFR3). However, only VEGFR1 and VEGFR2 are shown to be directly involved in VEGF binding $[16,17]$.

Single nucleotide polymorphisms (SNPs) are described in the VEGF gene, as well as in VEGFR1 and VEGFR2. For some of these, a direct association with the transcriptional activity of the corresponding genes has been shown [18-20]. Polymorphisms in the VEGFR2 promoter region have been found to influence the transcription activity of VEGFR2, contributing to the development of coronary artery lesions (CAL) in patients with Kawasaki disease (KD) and various cancers [21-23].

In light of recent interest in the interplay between decreased serum concentration of soluble VEGF (sVEGF) receptors and an increased serum concentration of VEGF, our group hypothesized that the development of OHSS is based on the finding that the higher the concentration of VEGF and the lower the concentration of the sVEGFreceptors, the higher the probability of the occurrence of OHSS is [11].

\section{Materials and methods}

In this case-control study, we included 116 women who suffered from severe OHSS during their IVF/ICSI therapy, and 124 healthy pregnant women who had delivered children in the department of OB/GYN at the General Hospital of Vienna (AKH). Institutional Review Board approval was obtained for the study at the MUW Ethikkommission (Reg. number 1398/2012).

We included all inpatients with the diagnosis of severe OHSS who required immediate hospitalization. The criteria for admission was met when at least two of the following factors were observed: 1) clinically relevant symptoms, such as diffuse abdominal discomfort, nausea, vomiting, dyspnea and tachypnea, subjective decreased urinary output; 2) electrolyte imbalance, leukocytosis $>20,000 / \mathrm{mm} 3$, creatinine $>1 \mathrm{mg} / \mathrm{dL}$, and hematocrit $>45 \%$; and 3 ) sonographically detectable free fluid (ascites and/or pleural effusion), with a significant increase in ovarian diameter $(>12 \mathrm{~cm})$; and 3) clinical signs, such as oliguria $<600 \mathrm{~mL}$ in 24 hours and hypotension.

Patients received stimulation with recombinant or human menopausal gonadotropins (hMG) after down-regulation via GnRHa (gonadotropin releasing hormone agonist) in case of a long protocol, or using Ganirelix (Orgalutran MSD Pharma) or Cetrorelix (Cetrotide MerckSerono) in case of an antagonist protocol.

All subjects received either 10,000 IU of hCG (Pregnyl, MSD Pharma), Choriogonadotropin alpha $250 \mu \mathrm{g}$ (Ovitrell MerckSerono), or Triptorelin acetate $0.2 \mathrm{mg}$ (Decapeptyl Ferring Austria) to trigger final oocyte maturation. Patients with any systemic disease were excluded from the study group and the control group. EDTA blood was obtained from all participants by peripheral antecubital vein puncture, and stored at $-18^{\circ} \mathrm{C}$ until the analysis was performed.

\section{Standardized OHSS treatment}

Once the patients were hospitalized with the diagnosis of OHSS III-IV according to Golan et al., they all received the same therapeutic protocol. Complete blood count with hematocrit, serum analysis of albumin, blood creatinine, blood urea, and creatinine clearance, liver function parameters, and coagulation tests on a daily basis were obtained in all patients. The amount of excreted urine, fluid balance, body weight, and abdominal circumference were carefully checked every 24 hours.

All patients were treated with 1000 ml lactated Ringer's solution twice a day, with furosemide $20 \mathrm{mg}$ (Lasix ${ }^{\odot}$ $20 \mathrm{mg}$, Sanofi, Vienna, Austria) to maintain the diuresis. Plasma volume expansion with hydroxyethyl starch solution 6\% (HAES-steril ${ }^{\circledR}$ 6\% Infusionslösung, $250 \mathrm{ml} \mathrm{KABI}$, Bd Homburg Germany), and/or human albumin 25\% (Human Albumin ${ }^{\oplus}$ Octapharm, Langfeld, Germany) were given once daily in cases of hypoalbuminemia or right after paracentesis $(>34 \mathrm{~g} / \mathrm{L})$.

If patients complained of pain or compromised pulmonary function, paracentesis was performed with a transvaginal approach.

Patients had to wear full-length venous support stockings. Prophylactic Enoxaparin therapy of $40 \mathrm{mg}$ on a daily basis (Lovenox $40 \mathrm{mg}$, Sanofi-Aventis GmbH Vienna) was given to all patients during in-patient treatment. The prophylactic therapy with Cabergoline o.5 (Dostinex ${ }^{\odot}$, Pharmacia, Berlin, Germany) was given to all patients from January 2008, orally once a day within eight days after retrieval.

The duration of the hospitalization was dependent on two factors: i) resolution of the symptoms; and ii) normalization of the lab results. Patients were discharged from the hospital when their hematocrit, without receiving any infusions, remained under $40 \%$.

\section{Genotyping}

We determined the genotypes of five polymorphisms in three genes by polymerase chain reaction and restriction fragment polymorphism analysis: VEGFR1 (other name FLT1); VEGFR2 (other name KDR); and VEGF. We used the SNP rs111458691 for genotyping the VEGFR1-519 polymorphism, rs2071559, rs2305948, and rs1870377 for genotyping the VEGFR2-604, VEGFR2-1192 and VEGFR21719 polymorphisms, respectively, and rs2010963 for genotyping the VEGF-405 polymorphism.

To determine the genotypes, we amplified a genomic DNA fragment by polymerase chain reaction (PCR). PCR amplification was performed with $0.2 \mathrm{mM}$ dNTP, $10 \mu \mathrm{M}$ forward primer, $10 \mu \mathrm{M}$ reverse primer, $1.5 \mathrm{mM}$ $\mathrm{MgCl} 2$, and $1 \mathrm{U}$ Taq polymerase (Sigma Qiagen, Hilden, Germany) in the recommended buffer. Cycling conditions were: 45 cycles in an Eppendorf Thermocycler at $94^{\circ} \mathrm{C}$ for $30 \mathrm{~s}, 60^{\circ} \mathrm{C}$ to $65^{\circ} \mathrm{C}$ for $30 \mathrm{~s}$, and $72^{\circ} \mathrm{C}$ for $45 \mathrm{~s}$ after the initial denaturation step $\left(94^{\circ} \mathrm{C}\right.$ for $\left.5 \mathrm{~min}\right)$. A 
seven-minute final extension step at $72^{\circ} \mathrm{C}$ was added. The total volume of the PCR reaction was $25 \mu \mathrm{l}$. The size of the PCR products, the enzymes used, and the resulting fragment length are given in Table 1. Fragments were visualized after electrophoresis on a $3 \%-4 \%$ agarose gel by staining with ethidium bromide. Pictures were taken with a Canon digital camera system (UVP Laboratory Products, USA).

\section{Statistical analysis}

Statistical analyses were performed using a chi-square test of association and a Fisher's exact probability test at the Vassar Stats homepage (http://vassarstats.net/). A value of $\mathrm{P}<0.05$ was defined as statistically significant. Odds ratios, confidence intervals, and linkage disequilibrium analyses were performed with $\mathrm{R}$, an open-source language and environment for statistical computing [24]. To adjust the values for multiple comparisons, we used the Benjamini-Hochberg correction method, which controls for false discovery rate (FDR) [25].

\section{Results}

Blood samples from 240 subjects (116 patients and 124 controls) were analyzed. An overrepresentation of the T allele of the VEGFR1-519 C > T polymorphism (rs111458691) was found in OHSS patients. The significant difference in the allele frequencies resulted in a 3.6-fold overrepresentation ( $\mathrm{p}=0.018)$, OR:3.62; CI: 1.16 - 11.27)). Although it did not reach a level of significance, a tendency toward overrepresentation of the A Allele of VEGFR2-1192 (rs2305948) in OHSS patients was also found $(p=0.06$, OR:1.76; CI: 0.97 - 3.18). The allele frequencies of VEGFR2-604 (rs2071559), VEGFR2-1719 (rs1870377), and VEGFA-405 (rs2010963) were not statistically different (Table 2).

We calculated different genotype models and considered the model with the smallest probability value as the best-fitting model for the respective genotype. Using an overdominant genotype model, we found that VEGF-405 polymorphism is significantly associated with OHSS ( $\mathrm{p}=0.000005$; OR:0.29; CI: $(0.17-0.50)$, and we found a significant association of VEGFR1-519 SNP with OHSS ( $\mathrm{p}=0.014$; OR: 3.79; CI: 1.2 - 11.97), using a co-dominant genotype model.

No significant difference in genotype distribution models was found for VEGFR2-604, VEGFR2-1192, and VEGFR21719 (Table 3).

The data from the three SNPs of VEGFR2 were tested for redundancy by linkage disequilibrium (LD) analyses. This analysis showed a significant LD for SNP VEGFR2604 vs. VEGFR2-1192 ( $\mathrm{D}^{\prime}=0,44 ; \mathrm{r}=-0.15 ; \mathrm{p}=0.001$ ) and for VEGFR2-1192 vs. VEGFR2-1719 (D'=0.31; $\mathrm{r}=0.19$; $\mathrm{p}=0.00003)$. However, this did not reveal a significant correlation between VEGFR2-604 and VEGFR2-1719.

Since we followed a standardized therapy for all our patients, we defined the day of discharge from the hospital as the day when the symptoms of OHSS were resolved. In Table 4, we examined the correlation between the duration of the hospitalization between the patients with and without the investigated polymorphisms. Therefore, we grouped patients into two groups with regard to their stay in our clinic (Group 1: one to nine days; group 2: 10 to 28 days; mean of all samples: 10.03 days). This subgroup analysis showed no statistically significant difference between patients who stayed longer than nine days in the hospital and patients who stayed between one and nine days in our clinic for every analyzed SNP.

\section{Discussion}

In this study, we investigated the association of five polymorphisms in three genes of the VEGF/VEGF receptor system and the occurrence of OHSS in 116 patients who suffered from OHSS and 124 healthy pregnant women. We analyzed a more general group of patients as controls, namely, healthy pregnant women, because this group represents a cross-sectional control compared to IVF patients, and is not selected by various fertility-related pathologies that are characteristic of IVF patients.

Table 1 Primers, fragment length and enzymes used for RFLP analysis

\begin{tabular}{|c|c|c|c|c|}
\hline Gene/SNP & Primers $\left(5^{\prime}-3^{\prime}\right)$ & Fragment lengths (bp) & Enzyme & RefSNP \\
\hline \multirow[t]{2}{*}{ VEGFR2- 604} & Fw: CAAACTTTCACTAGGGCTCTTCGT & $290 ; 174 ; 116$ & Bsml & 2071559 \\
\hline & Rev: AGCCACAAGGGAGAAGCGGATA & & & \\
\hline \multirow[t]{2}{*}{ VEGFR2-1192 } & Fw: TGAGGTTAAAAGTTCTGGTGTCCCTGTT & $262 ; 232 ; 30$ & BstZ17l & 2305948 \\
\hline & Rev: AATGTACAATCCTTGGTCACTCCGGGGTA & & & \\
\hline \multirow[t]{2}{*}{ VEGFR2-1719 } & Fw: CCTCCTGTATCCTGAATGAATCT & $404 ; 191 ; 213$ & Alul & 1870377 \\
\hline & Rev: GCCTCACATATTATTGTACCATCC & & & \\
\hline \multirow[t]{2}{*}{ VEGF-405 } & Fw: ATTTATTTTGCTTGCCATT & $304 ; 191 ; 213$ & BsmF1 & 2010963 \\
\hline & Rev: GTCTGTCTGTCTGTCCGTCA & & & \\
\hline \multirow[t]{2}{*}{ VEGFR1-519 } & Fw: GTGGCAACTTTGGGTTACCCA & $665 ; 520 ; 145$ & Nspl & 11145869 \\
\hline & Rev: CCTGACCCCTTCAGACTGTCC & & & \\
\hline
\end{tabular}


Table 2 Allele frequencies

\begin{tabular}{|c|c|c|c|c|c|}
\hline & OHSS (\%) & Control (\%) & P-value $^{a}$ & Chi & OR (Cl) \\
\hline \multicolumn{6}{|l|}{ VEGFR1-519 } \\
\hline C & $219(94.40)$ & 244 (98.39) & & & \\
\hline $\mathrm{T}$ & $13(5.60)$ & $4(1.61)$ & $0.018^{*}$ & 5.59 & $3.62(1.16-11.27)$ \\
\hline \multicolumn{6}{|l|}{ VEGFR2-604 } \\
\hline $\mathrm{T}$ & $107(46.12)$ & $125(50.40)$ & & & \\
\hline C & 125 (53.88) & $123(49.60)$ & 0.35 & 0.88 & $1.19(0.83-1.7)$ \\
\hline \multicolumn{6}{|l|}{ VEGFR2-1192 } \\
\hline G & 201 (86.64) & 228 (91.94) & & & \\
\hline A & 31 (13.36) & $20(8.06)$ & 0.06 & 5.59 & $1.76(0.97-3.18)$ \\
\hline \multicolumn{6}{|l|}{ VEGFR2-1719 } \\
\hline $\mathrm{T}$ & $55(23.71)$ & 60 (24.19) & & & \\
\hline A & 177 (67.29) & $188(75.81)$ & 0.88 & 0.02 & $0.97(0.64-1.48)$ \\
\hline \multicolumn{6}{|l|}{ VEGF-405 } \\
\hline G & 91 (39.22) & $108(43.55)$ & & & \\
\hline A & $141(60.78)$ & 140 (5645) & 0.34 & 0.92 & $0.84(0.58-1.20)$ \\
\hline
\end{tabular}

${ }^{a}$ Chi square test was used, with $p<0.05$ considered significant and marked with an asterisk. To adjust the values for multiple comparisons, we used the Benjamini-Hochberg $(\mathrm{BH})$ correction method, which controls false discovery rate (FDR).

Table 3 Genotypes

\begin{tabular}{llllllll}
\hline & Control & $\%$ & Patient & $\%$ & OR & Lower & Upper $^{\text {p-value }}$ \\
\hline VEGFR1-519 & & & & & & & \\
(Co-dominant) & 120 & 96,8 & 103 & 88,8 & 1,00 & - & - \\
C/C & 4 & 3,2 & 13 & 11,2 & 3,79 & 1,20 & 11,97 \\
$C / T$ & 0 & - & 0 & - & - & - & - \\
T/T & & & & & & &
\end{tabular}

(Co-dominant)

\begin{tabular}{|c|c|c|c|c|c|c|c|c|}
\hline C/C & 22 & 17,7 & 28 & 24,1 & 1 & - & - & 0,47 \\
\hline $\mathrm{C} / \mathrm{T}$ & 79 & 63,7 & 69 & 59,5 & 0,69 & 0,36 & 1,31 & \\
\hline $\mathrm{T} / \mathrm{T}$ & 23 & 18,5 & 19 & 16,4 & 0,65 & 0,28 & 1,48 & \\
\hline
\end{tabular}

\section{VEGFR2-1192}

(Co-dominant)

\begin{tabular}{llllllll} 
G/G & 106 & 85,5 & 90 & 77,6 & 1 & - & - \\
G/A & 16 & 12,9 & 21 & 18,1 & 1,55 & 0,76 & 3,14 \\
A/A & 2 & 1,6 & 5 & 4,3 & 2,94 & 0,56 & 15,54 \\
\hline
\end{tabular}

\section{VEGFR2-1719}

(Co-dominant)

\begin{tabular}{|c|c|c|c|c|c|c|c|c|}
\hline $\mathrm{A} / \mathrm{A}$ & 69 & 55,6 & 66 & 56,9 & 1 & - & - & 0,97 \\
\hline $\mathrm{A} / \mathrm{T}$ & 50 & 40,3 & 45 & 38,8 & 0,94 & 0,56 & 1,59 & \\
\hline $\mathrm{T} / \mathrm{T}$ & 5 & 4,0 & 5 & 4,3 & 1,05 & 0,29 & 3,78 & \\
\hline \multicolumn{9}{|c|}{ VEGF-405 } \\
\hline \multicolumn{9}{|c|}{ (Recessive) } \\
\hline $\mathrm{A} / \mathrm{A}-\mathrm{A} / \mathrm{G}$ & 116 & 93,5 & 97 & 83,6 & 1 & - & - & $0,01^{*}$ \\
\hline $\mathrm{G} / \mathrm{G}$ & 8 & 6,5 & 19 & 16,4 & 2,84 & 1,19 & 6,77 & \\
\hline
\end{tabular}

${ }^{a}$ Chi square test was used, with $p<0.05$ considered significant and marked with an asterisk. 
Table 4 Subgroup analysis

\begin{tabular}{|c|c|c|c|c|c|c|}
\hline \multicolumn{7}{|c|}{ Allele frequencies in accordance to hospital stay } \\
\hline & & OHSS-S (\%) & OHSS-L (\%) & P-value & Chi & OR (Cl) \\
\hline Mean (Range) & & $6.58(2-9)$ & $13.36(10-28)$ & & & \\
\hline \multirow[t]{2}{*}{ VEGFR1-519 } & C & $107(93.9 \%)$ & $112(94.9 \%)$ & & & \\
\hline & T & $7(6.1 \%)$ & $6(5.1 \%)$ & 0.78 & 0.73 & $1.22(0.39-3.75)$ \\
\hline \multirow[t]{2}{*}{ VEGFR2-604 } & $\mathrm{T}$ & $56(49.1 \%)$ & $51(43.2 \%)$ & & & \\
\hline & C & $58(51.9 \%)$ & $67(56.8 \%)$ & 0.79 & 0.78 & $0.93(0.55-1.57)$ \\
\hline \multirow[t]{2}{*}{ VEGFR2-1192 } & G & $99(86.8 \%)$ & $102(86.4 \%)$ & & & \\
\hline & A & $15(13.2 \%)$ & $16(13.6 \%)$ & 1.0 & 0.92 & $0.97(0.45-2.1)$ \\
\hline \multirow[t]{2}{*}{ VEGFr2-1719 } & T & $25(21.9 \%)$ & $30(25.4 \%)$ & & & \\
\hline & A & $89(78.1 \%)$ & $88(74.6 \%)$ & 0.54 & 0.53 & $1.22(0.66-2.23)$ \\
\hline \multirow[t]{2}{*}{ VEGF-405 } & G & 42 (36.8\%) & 49 (41.5\%) & & & \\
\hline & $A$ & $72(63.2 \%)$ & 69 (58.5\%) & 0.50 & 0.47 & $1.22(0.72-2.06)$ \\
\hline
\end{tabular}

Legend: Allel frequencies and $\mathrm{p}$ values are given for association of SNPs and duration time in hospital. OHSS-S: Duration in hospital 1-9 days, OHSS-L: Duration in hospital 10-28 days.

We found a significant association of the VEGF405 polymorphism (rs2010963) and the VEGFR1-519 polymorphism (rs111458691) with the occurrence of OHSS. However, the absolute difference of $5.6 \%$ and $1.6 \%$ in VEGFR1-519 T SNP for patients with OHSS shows that this SNP seems to be a rather rare allele.

The finding of a statistically significant association of the VEGF-405 SNP and OHSS development confirms and extends previous findings by Hanevik et al., who demonstrated an association of this SNP and OHSS in a study group of 53 Norwegian patients suffering from OHSS and a control group that performed IVF therapy but did not suffer from this complication [26]. Our collective comprised 116 OHSS patients with a presumably different Caucasian ethnicity than in Hanevik's study, and were compared to healthy pregnant women. Although our findings might strengthen the validity of a correlation between OHSS and the VEGF-405 genotype, these findings also open the possibility of a correlation between other fertilityrelated pathologies (e.g., PCOS, endometriosis) with this SNP that require IVF therapy. In both cases, this genotype variation might be of biological significance because it has been shown by other authors that this genotype contributes to VEGF serum levels [20] and that VEGF serum levels are associated with OHSS, PCOS, and endometriosis [27-29]. In addition, Watson et al. identified a predicted myeloid zinc finger binding site (MZF1) at polymorphism VEGF-405 that opens the possibility that variations at this site directly affect VEGF gene expression through a different binding efficiency of zinc finger transcription factors at its recognition site [18].

To the best of our knowledge, we show, for the first time, an association between the VEGF-receptor polymorphism VEGFR1-519 (rs111458691) and the occurrence of OHSS.
Previously, polymorphisms in the VEGF receptors were shown to be associated with some common cancers, stroke, systemic lupus erythematosus, and recurrent pregnancy loss [23,30-33]. Moreover, in colon and rectal cancer, it was suggested that the VEGF receptors have a greater influence on cancer risk than VEGF, and it is thought that this effect might be caused by an interaction of the VEGF receptors with inflammatory signals [23].

An analysis of the VEGFR1-519 SNP by Menendez et al. demonstrated that the T-allele generates a p53 response element, placing the VEGF-VEGF receptor system directly in the p53 stress response transcriptional network [34]. In our study, we found an overrepresentation of the T-allele in OHSS patients. This opens the intriguing possibility that p53-mediated stress signalling indirectly contributes to the pathology of OHSS, and led us to suggest a role for p53 in the pathology of OHSS. This idea is strengthened by the recent findings of Boudjenah et al., showing that p53 polymorphisms could influence the ovarian response to $\mathrm{rFSH}$ stimulation for patients undergoing an intracytoplasmic sperm injection program [35]. In a subgroup analysis of the OHSS patient group, we correlated the hospital stay with the various SNPs. This analysis revealed no significant differences in allele frequencies between patients who have a short stay in the hospital (one-to-nine days) and patients who required a longer habitation (1028 days). This finding suggests that the severity of OHSS based on hospital stay is not associated with any of the analyzed polymorphisms.

Our study has some limitations. This is a retrospective study and some data, such as full infertility anamnesis, possible medication effects, and the exact IVF protocolincluding E2 concentration on the day of retrieval-are missing because patients were included from other clinics after their OHSS symptoms required medical care in a 
specialized center. In addition, we found that the polymorphism VEGF-405 is out of the Hardy Weinberg equilibrium (HWE). Based on the small amount of possible further information available about our study population, a selection bias could not be excluded.

\section{Conclusion}

In the present study, we found a significant association of the VEGF405 polymorphism (rs2010963) and the VEGFR1519 polymorphism (rs111458691) with the occurrence of OHSS. The identification of a context between OHSS and specific gene variants of the VEGF/VEGF receptor system involved in vascular permeability enabled further insights concerning the pathophysiology and etiology of OHSS, and may contribute to a genetic characterization of susceptible women before beginning an ART program. Although the present study may provide informative and supportive data relating to the clinical significance of OHSS and its relationship to the VEGF/VEGF-receptor system, future larger studies should be performed to fully explore this putative gene-epidemiology interaction.

\section{Competing interests}

The authors declare no competing interests.

None of the authors have any relevant financial, personal, political, or religious interest linked to the subject of this article.

\section{Authors' contributions}

KN contributed to patient recruiting, interpretation of data, and manuscript writing and editing; PH and LS performed data collection and data mining, M Sch and CS contributed to data analysis, interpretation of data, and manuscript editing. MS contributed to patient recruiting and correcting the manuscript. DP analyzed and interpreted the data, contributed to manuscript writing and editing, and supervised the project. All authors read and approved the final manuscript.

\section{Acknowledgment}

We thank our patients and gratefully acknowledge the help of the members of the IVF Unit and the nurses in the patient care unit at the Women's Hospital in Vienna.

\section{Author details}

'Department of Endocrinology and Reproductive Medicine, Medical University Vienna, Währinger Gürtel 18-20, 1090 Vienna, Austria. ${ }^{2}$ Privatklinik Doebling, Fertility Center Doebling, Vienna, Austria.

Received: 26 January 2014 Accepted: 5 May 2014

Published: 13 May 2014

\section{References}

1. Golan A, Ron-el R, Herman A, Soffer Y, Weinraub Z, Caspi E: Ovarian hyperstimulation syndrome: an update review. Obstet Gynecol Surv 1989, 44(6):430-440.

2. Soares SR, Troncoso C, Bosch E, Serra V, Simon C, Remohi J, Pellicer A: Age and uterine receptiveness: predicting the outcome of oocyte donation cycles. J Clin Endocrinol Metab 2005, 90(7):4399-4404.

3. Orvieto R: Can we eliminate severe ovarian hyperstimulation syndrome? Hum Reprod 2005, 20(2):320-322.

4. Herr D, Sallmann A, Bekes I, Konrad R, Holzheu I, Kreienberg R, Wulff C: VEGF induces ascites in ovarian cancer patients via increasing peritoneal permeability by downregulation of Claudin 5. Gynecol Oncol 2012, 127(1):210-216.

5. Pietrowski D, Keck C: Differential regulation of ANG2 and VEGF-A in human granulosa lutein cells by choriogonadotropin. Exp Clin Endocrinol Diabetes 2004, 112(4):208-214.
6. Walz A, Keck C, Weber H, Kissel C, Pietrowski D: Effects of luteinizing hormone and human chorionic gonadotropin on corpus luteum cells in a spheroid cell culture system. Mol Reprod Dev 2005, 72(1):98-104.

7. Kasum M: New insights in mechanisms for development of ovarian hyperstimulation syndrome. Coll Antropol 2010, 34(3):1139-1143.

8. Abramov Y, Barak V, Nisman B, Schenker JG: Vascular endothelial growth factor plasma levels correlate to the clinical picture in severe ovarian hyperstimulation syndrome. Fertil Steril 1997, 67(2):261-265.

9. Artini PG, Fasciani A, Monti M, Luisi S, D'Ambrogio G, Genazzani AR: Changes in vascular endothelial growth factor levels and the risk of ovarian hyperstimulation syndrome in women enrolled in an in vitro fertilization program. Fertil Steril 1998, 70(3):560-564.

10. Agrawal R, Conway GS, Engmann L, Bekir JS, Jacobs HS, Sladkevicius P, Bekir J, Campbell S, Tan SL, Hoveyda F, Steele J, Lopez Bernal A, Barlow DH, Siano L, Schmidt D, Nulsen J, Maier D, Benadiva C: The pattern of changes in ovarian stromal and uterine artery blood flow velocities during in vitro fertilization treatment and its relationship with outcome of the cycle. Gynecol Endocrinol 1999, 13(1):26-33.

11. Pietrowski D, Szabo L, Sator M, Just A, Egarter C: Ovarian hyperstimulation syndrome is correlated with a reduction of soluble VEGF receptor protein level and a higher amount of VEGF-A. Hum Reprod 2012, 27(1):196-199.

12. Wang TH, Horng SG, Chang CL, Wu HM, Tsai YJ, Wang HS, Soong YK: Human chorionic gonadotropin-induced ovarian hyperstimulation syndrome is associated with up-regulation of vascular endothelial growth factor. I Clin Endocrinol Metab 2002, 87(7):3300-3308.

13. Raziel A, Friedler S, Schachter M, Strassburger D, Mordechai E, Ron-El R: Increased early pregnancy loss in IVF patients with severe ovarian hyperstimulation syndrome. Hum Reprod 2002, 17(1):107-110.

14. Shi W, Zhang S, Zhao W, Xia X, Wang M, Wang H, Bai H, Shi J: Factors related to clinical pregnancy after vitrified-warmed embryo transfer: a retrospective and multivariate logistic regression analysis of 2313 transfer cycles. Hum Reprod 2013, 28(7):1768-1775.

15. Griesinger G, Diedrich K, Devroey P, Kolibianakis EM: GnRH agonist for triggering final oocyte maturation in the $\mathrm{GnRH}$ antagonist ovarian hyperstimulation protocol: a systematic review and meta-analysis. Hum Reprod Update 2006, 12(2):159-168.

16. Neufeld G, Cohen T, Gengrinovitch S, Poltorak Z: Vascular endothelial growth factor (VEGF) and its receptors. FASEB J 1999, 13(1):9-22.

17. Ferrara $\mathrm{N}$ : Vascular endothelial growth factor: basic science and clinical progress. Endocr Rev 2004, 25(4):581-611.

18. Watson CJ, Webb NJ, Bottomley MJ, Brenchley PE: Identification of polymorphisms within the vascular endothelial growth factor (VEGF) gene: correlation with variation in VEGF protein production. Cytokine 2000, 12(8):1232-1235.

19. Awata T, Inoue K, Kurihara S, Ohkubo T, Watanabe M, Inukai K, Inoue I, Katayama S: A common polymorphism in the 5'-untranslated region of the VEGF gene is associated with diabetic retinopathy in type 2 diabetes. Diabetes 2002, 51(5):1635-1639.

20. Steffensen KD, Waldstrom M, Brandslund I, Jakobsen A: The relationship of VEGF polymorphisms with serum VEGF levels and progression-free survival in patients with epithelial ovarian cancer. Gynecol Oncol 2010, 117(1):109-116.

21. Wang $Y$, Zheng $Y$, Zhang $W$, Yu H, Lou K, Zhang Y, Qin Q, Zhao B, Yang Y, Hui R: Polymorphisms of KDR gene are associated with coronary heart disease. J Am Coll Cardiol 2007, 50(8):760-767.

22. Kariyazono H, Ohno T, Khajoee V, Ihara K, Kusuhara K, Kinukawa N, Mizuno $Y$, Hara T: Association of vascular endothelial growth factor (VEGF) and VEGF receptor gene polymorphisms with coronary artery lesions of Kawasaki disease. Pediatr Res 2004, 56(6):953-959.

23. Slattery ML, Lundgreen A, Wolff RK: VEGFA, FLT1, KDR and colorectal cancer: assessment of disease risk, tumor molecular phenotype, and survival. Mol Carcinog 2014, 53 Suppl 1:E140-150.

24. Development Core Team R: R: A lanquage and environment for statistical computing 2009. Vienna, Austria: R Foundation for Statistical Computing; 2009.

25. Benjamini $Y$, Hochberg $Y$ : Controlling the false discovery rate - a practical and powerful approach to multiple testing. J R Stat SoC B Methodological 1995, 57(1):289-300.

26. Hanevik HI, Hilmarsen HT, Skjelbred CF, Tanbo T, Kahn JA: Increased risk of ovarian hyperstimulation syndrome following controlled ovarian 
hyperstimulation in patients with vascular endothelial growth factor +405 cc genotype. Gynecol Endocrinol 2012, 28(11):845-849.

27. Peitsidis $P$, Agrawal R: Role of vascular endothelial growth factor in women with PCO and PCOS: a systematic review. Reprod Biomed Online 2010, 20(4):444-452.

28. Li YZ, Wang LJ, Li X, Li SL, Wang JL, Wu ZH, Gong L, Zhang XD: Vascular endothelial growth factor gene polymorphisms contribute to the risk of endometriosis: an updated systematic review and meta-analysis of 14 case-control studies. Genet Mol Res 2013, 12(2):1035-1044.

29. Kianpour M, Nematbakhsh M, Ahmadi SM, Jafarzadeh M, Hajjarian M, Pezeshki Z, Safari T, Eshraghi-Jazi F: Serum and peritoneal fluid levels of vascular endothelial growth factor in women with endometriosis. Int J Fertil Steril 2013, 7(2):96-99.

30. Scartozzi M, Bianconi M, Faloppi L, Loretelli C, Bittoni A, Del Prete M, Giampieri R, Maccaroni E, Nicoletti S, Burattini L, Minardi D, Muzzonigro G, Montironi R, Cascinu S: VEGF and VEGFR polymorphisms affect clinical outcome in advanced renal cell carcinoma patients receiving first-line sunitinib. Br J Cancer 2013, 108(5):1126-1132.

31. Shibuya M: Vascular endothelial growth factor and its receptor system: physiological functions in angiogenesis and pathological roles in various diseases. J Biochem 2013, 153(1):13-19.

32. Su MT, Lin SH, Lee IW, Chen YC, Hsu CC, Pan HA, Kuo PL: Polymorphisms of endocrine gland-derived vascular endothelial growth factor gene and its receptor genes are associated with recurrent pregnancy loss. Hum Reprod 2010, 25(11):2923-2930.

33. Su MT, Lin SH, Lee IW, Chen YC, Kuo PL: Association of polymorphisms/ haplotypes of the genes encoding vascular endothelial growth factor and its KDR receptor with recurrent pregnancy loss. Hum Reprod 2011, 26(4):758-764

34. Menendez D, Krysiak O, Inga A, Krysiak B, Resnick MA, Schonfelder G: A SNP in the flt-1 promoter integrates the VEGF system into the p53 transcriptional network. Proc Natl Acad Sci U S A 2006, 103(5):1406-1411.

35. Boudjenah $\mathrm{R}$, Molina-Gomes $\mathrm{D}$, Torre A, Bergere M, Bailly M, Boitrelle F, Taieb S, Wainer R, Benahmed M, de Mazancourt P, Selva J, Vialard F: Genetic polymorphisms influence the ovarian response to $\mathrm{rFSH}$ stimulation in patients undergoing in vitro fertilization programs with ICSI. PLOS One 2012, 7(6):e38700.

doi:10.1186/1757-2215-7-54

Cite this article as: Nouri et al:: Polymorphisms of VEGF and VEGF receptors are associated with the occurrence of ovarian

hyperstimulation syndrome (OHSS) - a retrospective case-control study. Journal of Ovarian Research 2014 7:54.

\section{Submit your next manuscript to BioMed Central and take full advantage of:}

- Convenient online submission

- Thorough peer review

- No space constraints or color figure charges

- Immediate publication on acceptance

- Inclusion in PubMed, CAS, Scopus and Google Scholar

- Research which is freely available for redistribution 